CME

Open

\title{
The Association between Distal Findings and Proximal Colorectal Neoplasia: A Systematic Review and Meta-Analysis
}

Jason L.W. Huang, $\mathrm{MD}^{1,9}$, Y.H. Wang, $\mathrm{PhD}^{2,9}$, Johnny Y. Jiang, $\mathrm{PhD}^{3}$, C.P. Yu, $\mathrm{PhD}^{4}$, Y.L. Wu, $\mathrm{MD}^{5}$, P. Chen, MD ${ }^{5}$, X.Q. Yuan, MD Harry H.X. Wang, $\mathrm{PhD}^{6}$ and Martin C.S. Wong, $\mathrm{MD}^{1,7,8}$

OBJECTIVES: Whether screening participants with distal hyperplastic polyps (HPs) detected by flexible sigmoidoscopy (FS) should be followed by subsequent colonoscopy is controversial. We evaluated the association between distal HPs and proximal neoplasia (PN)/advanced proximal neoplasia (APN) in asymptomatic, average-risk patients.

METHODS: $\quad$ We searched Ovid Medline, EMBASE, and the Cochrane Library from inception to 30 June 2016 and included all screening studies that examined the relationship between different distal findings and PN/APN. Data were independently extracted by two reviewers with disagreements resolved by a third reviewer. We pooled absolute risks and odds ratios (ORs) with a random effects meta-analysis. Seven subgroup analyses were performed according to study characteristics. Heterogeneity was characterized with the $R^{2}$ statistics.

RESULTS: $\quad$ We analyzed 28 studies (104,961 subjects). When compared with normal distal findings, distal HP was not associated with PN (OR=1.16, 95\% confidence interval $\left.(\mathrm{Cl})=0.89-1.51, P=0.14, R^{2}=40 \%\right)$ or APN (OR=1.09, 95\% Cl=0.87-1.36, $\left.P=0.39, P^{2}=5 \%\right)$, while subjects with distal non-advanced or advanced adenoma had higher odds of PN/APN. Higher odds of PN/APN were observed for more severe distal lesions. Weaker association between distal and proximal findings was noticed in studies with higher quality, larger sample size, population-based design, and more stringent endoscopy quality-control measures. The Egger's regression tests showed all P>0.05.

CONCLUSIONS: Distal HP is not associated with PN/APN in asymptomatic screening population when compared with normal distal findings. Hence, the presence of distal HP alone detected by FS does not automatically indicate colonoscopy referral for all screening participants, as other risk factors of PN/APN should be considered.

SUPPLEMENTARY MATERIAL is linked to the online version of the paper at http://www.nature.com/ajg

Am J Gastroenterol 2017; 112:1234-1245; doi:10.1038/ajg.2017.130; published online 30 May 2017

\section{INTRODUCTION}

Screening for colorectal cancer (CRC) has been proven as an effective prevention strategy to reduce CRC incidence and mortality (1). Many international guidelines recommended CRC screening by flexible sigmoidoscopy (FS) on a 5-yearly basis, and colonoscopy were performed 10 yearly (2-4). FS has been demonstrated to reduce CRC mortality by randomized controlled trials and systematic reviews. In relatively resource-deprived countries where colonoscopic capacity may be limited, FS bears potential as a primary screening test as it can be performed by

${ }^{1}$ School of Public Health and Primary Care, Faculty of Medicine, Chinese University of Hong Kong, Hong Kong, China; ${ }^{2}$ School of Basic Medicine, Peking Union Medical College and Institute of Basic Medical Sciences, Chinese Academy of Medical Sciences, Beijing, China; ${ }^{3}$ Peking Union School of Public Health, Chinese Academy of Medical Sciences and Peking Union Medical College, Beijing, China; ${ }^{4}$ Li Ping Medical Library, Chinese University of Hong Kong, Hong Kong, China; ${ }^{5}$ North Ruijin Hospital, Shanghai Jiaotong University, Shanghai, China; ${ }^{6}$ School of Public Health, Sun Yat-sen University, Guangzhou, China; ${ }^{7}$ nstitute of Digestive

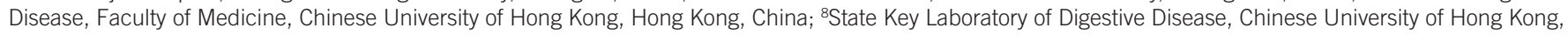
Hong Kong, China; ${ }^{9}$ These authors contributed as equal first authors. Correspondence: Martin C.S. Wong, MD, MPH, School of Public Health, Prince of Wales Hospital, Shatin, Hong Kong SAR, China. E-mail: wong_martin@cuhk.edu.hk

Received 7 November 2016; accepted 1 April 2017 
primary care practitioners (2). FS-based screening could examine neoplastic lesions in the distal colorectum. Any distal lesions detected could indicate synchronous risk of proximal neoplasia (PN) and advanced proximal neoplasia (APN). According to the latest US Preventive Services Task Force Recommendation Statements published in $2016(5,6)$, FS is one of the preferred tests of choice designed both to detect and prevent CRC if colonoscopy is not available or acceptable to patients.

Understanding the association between distal and proximal findings is clinically important, as it guides subsequent followup for subjects with distal lesions found on FS. In four published meta-analyses (7-10), the relationship between distal hyperplastic polyp (HP) and PN/APN in asymptomatic population presented mixed conclusions. Dave et al. (7) proposed that, for asymptomatic subjects, any distal HP detected by FS should be referred for colonoscopy workup due to an excessive $20-25 \%$ risk of any PN and $4-5 \%$ risk of APN. This conclusion was later challenged by findings from two meta-analyses $(8,9)$ that found no excessive risk of PN or APN conferred by the presence of distal HPs. However, the latest meta-analysis performed in 2012 that examined the relationship between distal lesions and PN/APN (10) concluded that all types of distal lesions, including HPs, were predictive of PN while all types of distal neoplasia were predictive of APN. Among these four meta-analyses, nevertheless, three were published more than a decade ago; and in the latest study published in 2012, approximately one-third of all the articles (12 in 40) selected were from symptomatic subjects, and hence its generalizability to guide CRC screening among asymptomatic subjects was limited. In addition, since year 2012, several studies with large sample size (11-16) were published and many of them were from population-based screening programs (13-15). These additional studies allow re-synthesis of existing data to evaluate the association between distal and proximal lesions.

The purpose of this systematic review and meta-analysis is to analyze all available data on the risk of PN and APN in asymptomatic subjects who were detected as having distal lesions with different types of histopathology. In particular, we tested the a priori hypothesis that distal HP was not associated with PN in asymptomatic screening populations, aiming to inform necessity of subsequent colonoscopy workup for individuals with distal HP detected by FS.

\section{METHODS}

\section{Search strategy and selection criteria}

The systematic review and meta-analysis adhered to the PRISMA (Preferred Reporting Items for Systematic reviews and metaAnalyses) statement (17), performed according to a predetermined protocol. We searched Ovid Medline (1946 to 30 June 2016), EMBASE (1976 to 30 June 2016), and the Cochrane Library (1988 to 31 May 2016). The search strategy was listed as below:

1. Rectal Neoplasms/or Colorectal Neoplasms/or Colonic Polyps/or Colonic Neoplasms/
2. (( (colon ${ }^{\star}$ or rectal or colorectal) adj (cancer ${ }^{\star}$ or neoplas ${ }^{\star}$ or tumor $^{*}$ or tumour ${ }^{*}$ or carcinoma ${ }^{*}$ or sarcoma ${ }^{*}$ or adenom ${ }^{*}$ or adeno? carcinoma ${ }^{*}$ or lesion ${ }^{*}$ or polyp $\left.{ }^{\star}\right)$ ) or CRC).ti,ab.

3. ((proximal or right-side ${ }^{\star}$ or "right side ${ }^{\star}$ ) ) adj2 (neoplas* or lesion ${ }^{*}$ or tumor* or tumour ${ }^{*}$ or polyp $\left.\left.{ }^{\star}\right)\right)$.mp.

4. ((distal or left-side* or "left side*") adj2 (neoplas* or lesion* $^{*}$ or tumor ${ }^{\star}$ or tumour* or polyp $\left.p^{*}\right)$.mp.

5. case control studies/ or cohort studies/or cross-sectional studies

6. ((cohort adj (study or studies)) or "case control" or "cohort analy $y^{\star \prime}$ or (observational adj (study or studies)) or longitudinal or retrospective or "cross sectional" or cross-sectional or (follow up adj (study or studies))).mp.

7. 1 or 2

8. 5 or 6

9. 3 and 4

10. 7 and 8

11. 9 and 10

We restricted our search to cross-sectional studies, case control studies, and prospective cohort studies on CRC screening that examined the relationship between distal findings of various histopathology and PN/APN for average-risk, asymptomatic subjects. The following types of studies were excluded:

1. Studies that recruited symptomatic patients $(18,19)$;

2. Studies that did not examine the association between distal and proximal findings (20);

3. Studies without data on PN or APN $(21,22)$;

4. Studies where the screening participants had high risk for CRC, such as those with positive family history (23);

5. Studies that consist of data on proximal advanced serrated lesions only (24).

We obtained data from summary estimates of all eligible studies without any language limitations. Reference lists of eligible studies and related meta-analyses were hand searched to identify further relevant studies.

\section{Data analysis}

Two reviewers (J.L.W.H., Y.H.W.) independently screened all abstracts identified in the initial search and excluded studies not fulfilling the eligible criteria. They extracted data from all selected full-text articles reviewed in duplicate, and in cases of disagreement, consensus was made via referral to a third reviewer (M.C.S.W.). The following variables were collected from each study: sample size, mean age of study participants, proportion of male subjects, research type (cross-sectional, case control, or cohort studies), endoscopic strategies (colonoscopy; sigmoidoscopy followed by colonoscopy, if necessary), and program design (population-based or opportunistic screening), as well as endoscopy quality-control measures (critical or normal).

In these studies, the odds ratio (OR) and absolute risk for PN or APN conferred by distal HP, distal adenoma (AD), or distal advanced neoplasia (AN), when compared with subjects with 
normal distal findings, were retrieved. The proportions of individuals with PN and APN in all eligible studies were also examined. The proportions of PN and APN were first synthesized and then examined in four types of subjects with various distal findings: normal, $H P, A D$, and $A N$. AN was defined as adenomas measuring $\geq 10 \mathrm{~cm}$, adenomas with villous portions, high-grade dysplasia, adenocarcinomas, or any combination thereof (20). If multiple lesions were reported in one subject, we used the most advanced distal or proximal lesion as the finding. The primary outcomes included the ORs and proportion of PN or APN among the subgroups of HP, AD, or $\mathrm{AN}$, as well as the respective $95 \%$ confidence interval (CI). The Mantel-Haenszel method based on a random-effects model was used. We tested for heterogeneity by calculating $P$ value and the $I^{2}$ statistic in a standard manner, where $I^{2}>50 \%$ or $P<0.05$ was considered as a threshold indicating significant heterogeneity.

We used the statistical analysis software (Revman 5.3, Copenhagen, Denmark: The Nordic Cochrane Centre, The Cochrane Collaboration, 2014) to synthesize the pooled estimation of OR and perform subgroup analysis. We explored potential publication bias with an inverted funnel plot analysis with Eggers' regression model by Comprehensive Meta Analysis (version 2.2, Biostat, 2011, Englewood, NJ).

\section{Subgroup analysis}

We conducted a comprehensive quality assessment for all selected studies during our review process. Because our selected articles are observational studies, we employed the Newcastle Ottawa Scale for cross-sectional studies (25) to evaluate the selection, comparability, and outcome among the studies. We renamed the index according to the above identified variables. Studies that reported compliance with endoscopic quality-control protocols were scored 2 while studies that did not report endoscopic quality were scored 1 . If there were no descriptions of the endoscopic tests, a zero score was assigned. For pathology reporting of colorectal findings, we assigned a score of 2 for blind reporting with universal pathological standard; a score of 1 for simple description of the reporting process, and 0 for studies giving no details. For subject selection, those studies collecting data based on population registries, enrolling subjects from predefined protocols, or GP rosters were given 1 point; while recruitment of patients from special populations or physician referral were given zero points, owing to the limited representativeness of the target population. Because the average sample size of our selected studies were $>3,000$, we named studies with $>3,000$ subjects as large population (score $=1$ ), and studies with sample size $<3,000$ as small population (score $=0$ ). Regarding statistical tests, if the tests used to analyze the data were described clearly and judged appropriately, and the measurement of the association was presented, including CIs and the probability level ( $P$ value), 1 point was given; otherwise, a zero point was assigned. If there was a control variable for APN in the study,

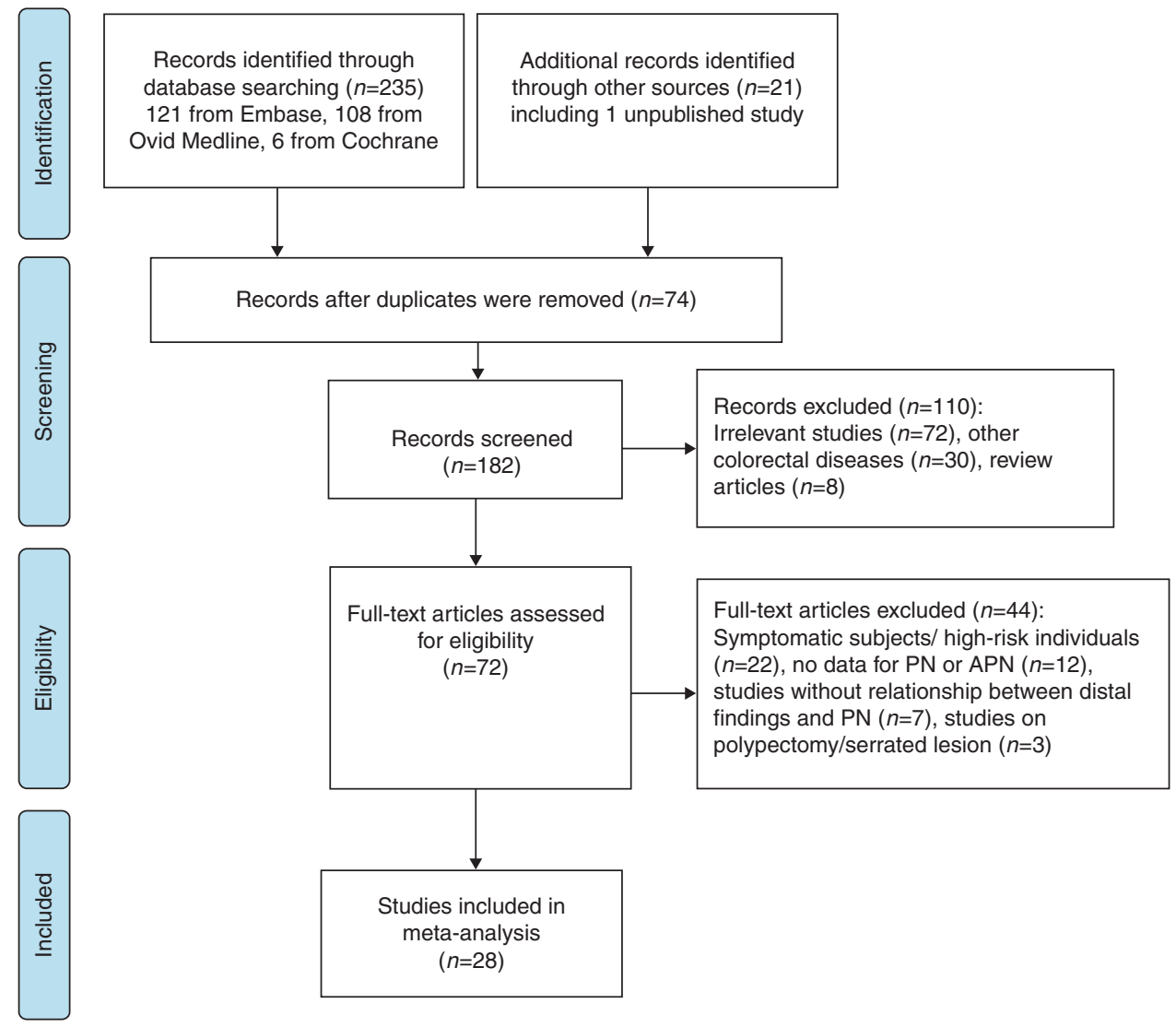

Figure 1. Results of the literature search. APN, advanced proximal neoplasia; PN, proximal neoplasia. 
耪离

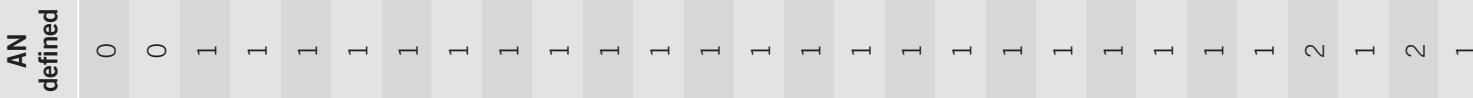

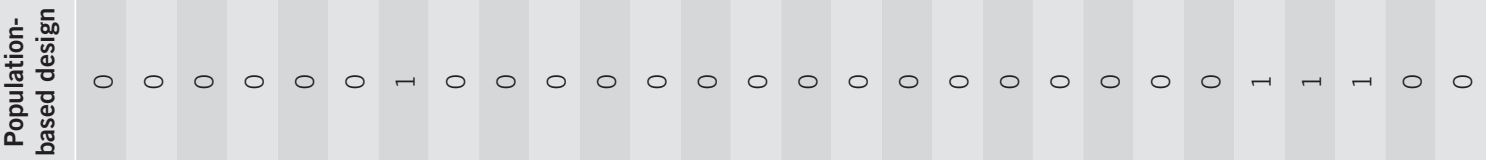

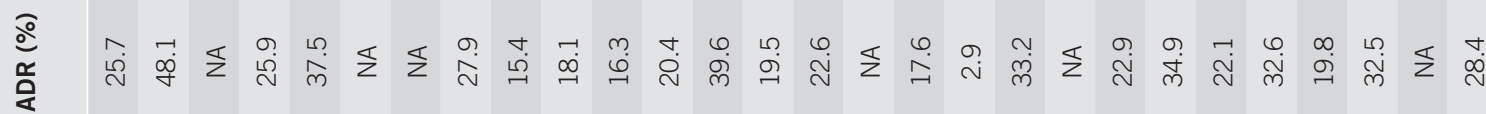

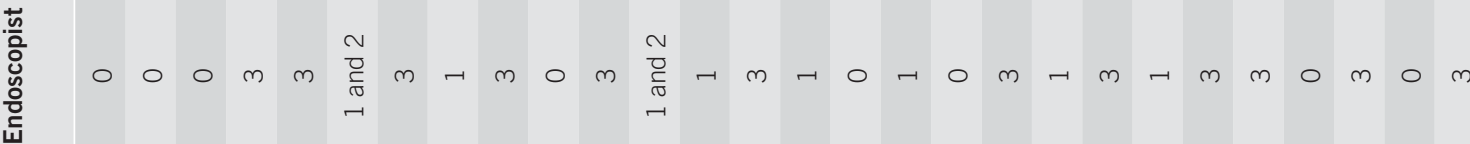

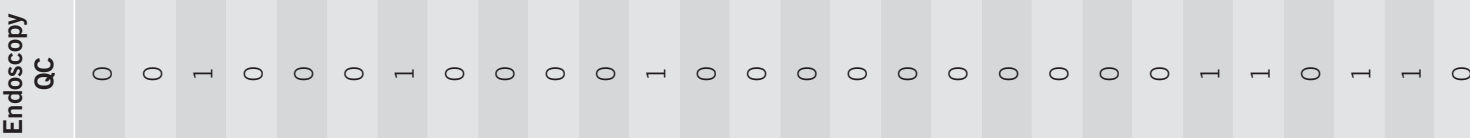

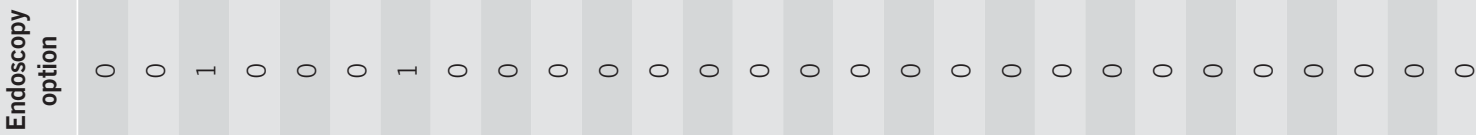

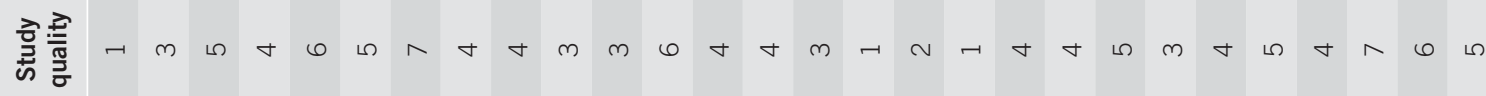

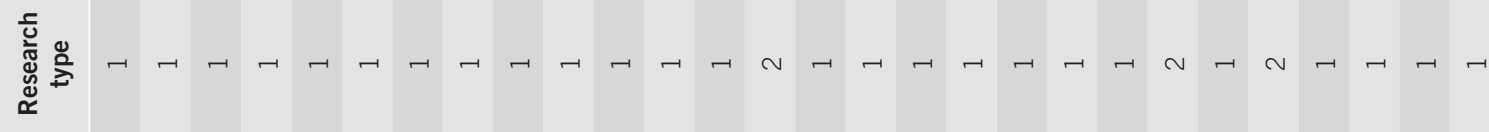

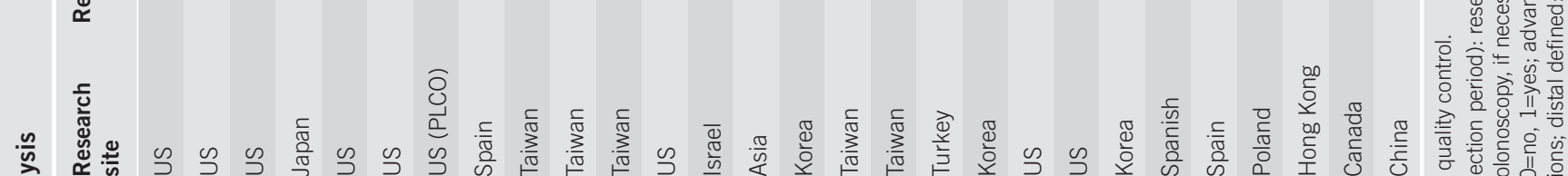

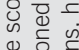
m

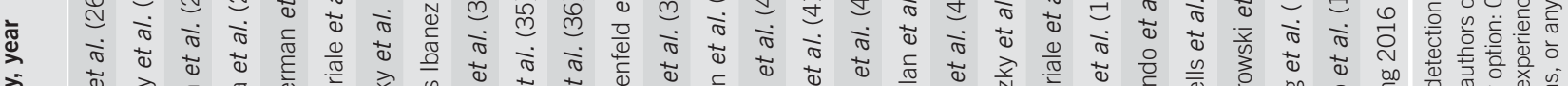

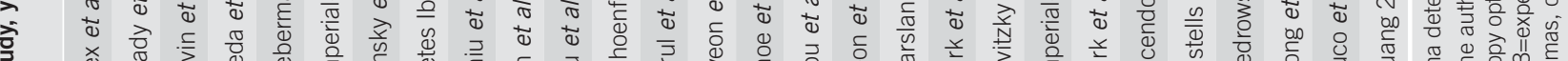
की 


\begin{tabular}{|c|c|c|c|}
\hline Year range of publication & 1990-1999 & 2000-2009 & 2010-2016 \\
\hline $\begin{array}{l}\text { Studies with large sample } \\
\operatorname{size}^{a}(\%, n / N)\end{array}$ & $0(0 / 3)$ & $43.8(7 / 16)$ & $88.9(8 / 9)$ \\
\hline $\begin{array}{l}\text { High-quality studies } \\
(\%, n / N)\end{array}$ & $33.3(1 / 3)$ & $62.5(10 / 16)$ & $88.9(8 / 9)$ \\
\hline $\begin{array}{l}\text { Population-based studies } \\
(\%, n / N)\end{array}$ & $0(0 / 3)$ & $6.3(1 / 16)$ & $33.3(3 / 9)$ \\
\hline
\end{tabular}

one extra mark was given. Thus there was a maximum of eight points for Newcastle Ottawa Scale assessment in this study. Again, two authors assessed all the selected studies separately and sought consensus for any disagreements through referral to the third reviewer.

The data were expected to be heterogeneous. Seven subgroup analyses on the risk of PN and APN were conducted according to the study characteristics: (1) study quality: high-quality score (4-8) vs. low-quality score (1-3); (2) sample size: $n>3000$ vs. $n \leq 3000$; (3) program design: population-based vs. opportunistic screening; (4) endoscopy quality control: normal procedures vs. high-level quality control; (5) the inclusion of serrated lesion in the definition of AN vs. not; (6) the definition of distal lesions: based on the splenic flexure as the demarcation point vs. the rectosigmoid; and (7) the procedure of examination: FS followed by a subsequent colonoscopy as a separate procedure vs. colonoscopy only. These subgroup analyses are important as we perceived them as potential effect modifiers of the present meta-analyses.

\section{RESULTS}

A total of 235 titles were obtained from the three databases (Figure 1), in addition to another 21 titles from previous systematic reviews and 1 unpublished study performed by our research group) (47). After excluding 74 duplicates, 182 abstracts were reviewed. Among them, 110 articles were excluded based on the selection criteria. After reviewing 72 full texts, 44 studies were found ineligible. Twenty-eight studies were finally included in the meta-analysis with a total of 104,961 subjects (Table 1), and the adenoma detection rate ranged from $2.9 \%$ to $48.1 \%$. The majority of selected studies that employed a population-based design in recruiting subjects were of high quality and used large sample size that were published after 2010 (Table 2).

From available data among the selected studies, the proportion of PN was $13.2 \%$ (95\% CI, 10.7-16.1\%) and that for APN was $2.2 \%$ (95\% CI, 1.7-2.8\%) (Figure 2a,b). Table 3 shows the pooled proportion and OR for the association between distal lesions and $\mathrm{PN} / \mathrm{APN}$. Among asymptomatic subjects, neither $\mathrm{PN}(\mathrm{OR}=1.16$, 95\% CI $\left.0.89-1.51, P=0.14, I^{2}=40 \%\right)$ nor APN (OR=1.09, 95\% CI 0.87-1.36, $\left.P=0.39, I^{2}=5 \%\right)$ was associated with distal HP when compared with those having normal distal colon. Very few studies reported that distal HP had higher odds of PN and APN, when compared with individuals with normal distal findings. Subjects with distal $\mathrm{AD}$ had significantly higher odds of $\mathrm{PN}(\mathrm{OR}=2.36,95 \%$ CI 1.91-2.92) and APN (OR=2.52, 95\% CI 1.84-3.46) compared with subjects with normal distal findings. These increased odds could also be observed in subjects with distal AN. It was found that the more advanced the distal lesions, the higher the odds of PN/APN. There was no significant heterogeneity when the associations between distal HP and PN $\left(I^{2}=40 \%, P=0.28\right) / \mathrm{APN}\left(I^{2}=5 \%\right.$, $P=0.39$ ) were examined (Figure 3a,b).

Table 4 shows subgroup analyses according to study characteristics that were regarded as potential moderators of the association between distal findings and PN/APN. Weaker associations were noticed in high-quality studies than in low-quality ones (AN-PN); in studies with large sample size than those with small sample size (AD-PN); in studies based on population-based design than those based on opportunistic screening approaches (AN-PN, AD-APN, AN-APN, HP-APN); in studies with critical endoscopy quality control than in studies with normal quality-control measures (AN-PN, AN-APN); in studies where distal lesions were defined as those located in rectosigmoid vs. studies where distal lesions were defined as those distal to the splenic flexure (AD-APN, AN-APN); in studies where FS was performed followed by colonoscopy as a separate procedure than in studies where only colonoscopy was performed (HP-PN, AD-PN, AD-APN, AN-PN, AN-APN). Supplementary Figure S1 illustrates the Egger's regression tests for publication bias. Except for distal AN-APN, all regression tests had $P$ values $>0.05$. The pooled prevalence of isolated PN $(5.6 \%$, 95\% CI 3.3-9.1\%) and isolated APN (1.0\%, 95\% CI 0.9-1.2\%) are shown in Supplementary Figure S2). The proportion of APN was $1.9 \%$ (95\% CI 1.5-2.5\%) among subjects with normal distal findings and 2.4\% (95\% CI 1.9-3.1\%) among subjects with distal HPs $(P=0.390)$.

\section{DISCUSSION}

This study found that distal HP was not associated with higher odds of APN or PN. The findings were robust from subgroup analyses with no publication biases detected. On the contrary, the presence of distal $\mathrm{AN}$ or $\mathrm{AD}$ were significantly associated with APN/PN. Whether to refer subjects with distal HP detected by FS for colonoscopy workup has been the subject of a long-lasting debate beginning in the 1980s-1990s, leading to three meta-analyses performed in the early 2000s. The study by Lin et al. (9) was the only evaluation that performed subgroup analysis stratifying 21 studies into screening and diagnostic cohorts. It was concluded that there was no increased risk of PN and APN in subjects with distal HP when compared with those having normal distal findings, based on observations in asymptomatic screening individuals. Two meta-analyses $(7,8)$ reported that in screening studies the relative risk of distal HP for PN (1.3, 95\% CI 0.9-1.8), the OR of distal HP for PN (1.44, 95\% CI 0.79-2.62), and the OR of distal HP for APN (1.63, 95\% CI 0.61-4.33) were not statistically significant, yet a recent meta-analysis found that $\mathrm{HP}$ was a predictor for PN (OR=1.8, 95\% CI 1.3-2.5) (10). Our study is consistent 
Study name

$\begin{array}{lc} & \text { Total } \\ & 42 / 162 \\ \text { Brady et al. (1993) } & 92 / 860 \\ \text { Byeon et al. (2007) } & 767 / 5059 \\ \text { Castells et al. (2013) } & 112 / 1708 \\ \text { Chiu et al. (2005) } & 570 / 5086 \\ \text { Choe et al. (2007) } & 14 / 1064 \\ \text { Erarslan et al. (2009) } & 405 / 3131 \\ \text { Ikeda et al. (2000) } & 667 / 2972 \\ \text { levin et al. (1999) } & 126 / 696 \\ \text { Lucendo et al. (2013) } & 288 / 3951 \\ \text { Park et al. (2009) } & 1679 / 6200 \\ \text { Park et al. (2012) } & 1681 / 8802 \\ \text { Pinsky et al. (2003) } & 89 / 482 \\ \text { Rex et al. (1992) } & 98 / 1382 \\ \text { Soon et al. (2008) } & 206 / 1177 \\ \text { Strul et al. (2006) } & 1073 / 5879 \\ \text { Wong et al. (2014) } & 7909 / 48611\end{array}$

b

Study name

Betes Ibanez et al. (2004)
Byeon et al. (2007)
Castells et al. (2013)
Chiu et al. (2005)
Choe et al. (2007)
Erarslan et al. (2009)
Huang et al. (2016)
Ikeda et al. (2000)
Imperiale et al. (2003)
Imperiale et al. (2012)
Kiedrowski et al. (2014)
levin et al. (1999)
Levitzky et al. (2011)
Lieberman et al. (2000)
Liou et al. (2007)
Liu et al. (2005)
Lucendo et al. (2013)
Park et al. (2009)
Park et al. (2012)
Pinsky et al. (2003)
Ruco et al. (2015)
Soon et al. (2008)
Wong et al. (2014)

Events/total

$\begin{array}{cc}\begin{array}{c}\text { Event } \\ \text { rate }\end{array} & \begin{array}{c}\text { Lower } \\ \text { limit }\end{array} \\ 0.259 & 0.198 \\ 0.107 & 0.088 \\ 0.152 & 0.142 \\ 0.066 & 0.055 \\ 0.112 & 0.104 \\ 0.013 & 0.008 \\ 0.129 & 0.118 \\ 0.224 & 0.210 \\ 0.181 & 0.154 \\ 0.073 & 0.065 \\ 0.271 & 0.260 \\ 0.191 & 0.183 \\ 0.185 & 0.152 \\ 0.071 & 0.059 \\ 0.175 & 0.154 \\ 0.183 & 0.173 \\ 0.132 & 0.107\end{array}$

Upper
limit
0.332
0.129
0.162
0.078
0.121
0.022
0.142
0.240
0.211
0.081
0.282
0.199
0.222
0.086
0.198
0.193
0.161

Event rate and $95 \% \mathrm{Cl}$

\section{Relative}

weight

5.7
6.2
6.5
6.3
6.5
4.9
6.5
6.5
6.3
6.4
6.5
6.5
6.2
6.2
6.4
6.5

$\mid$
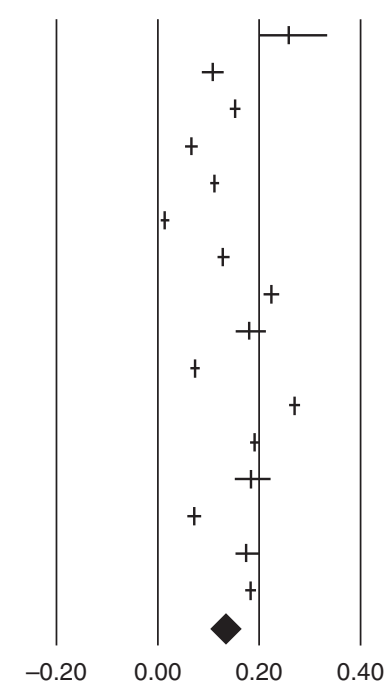

\section{Event rate and $95 \% \mathrm{Cl}$}

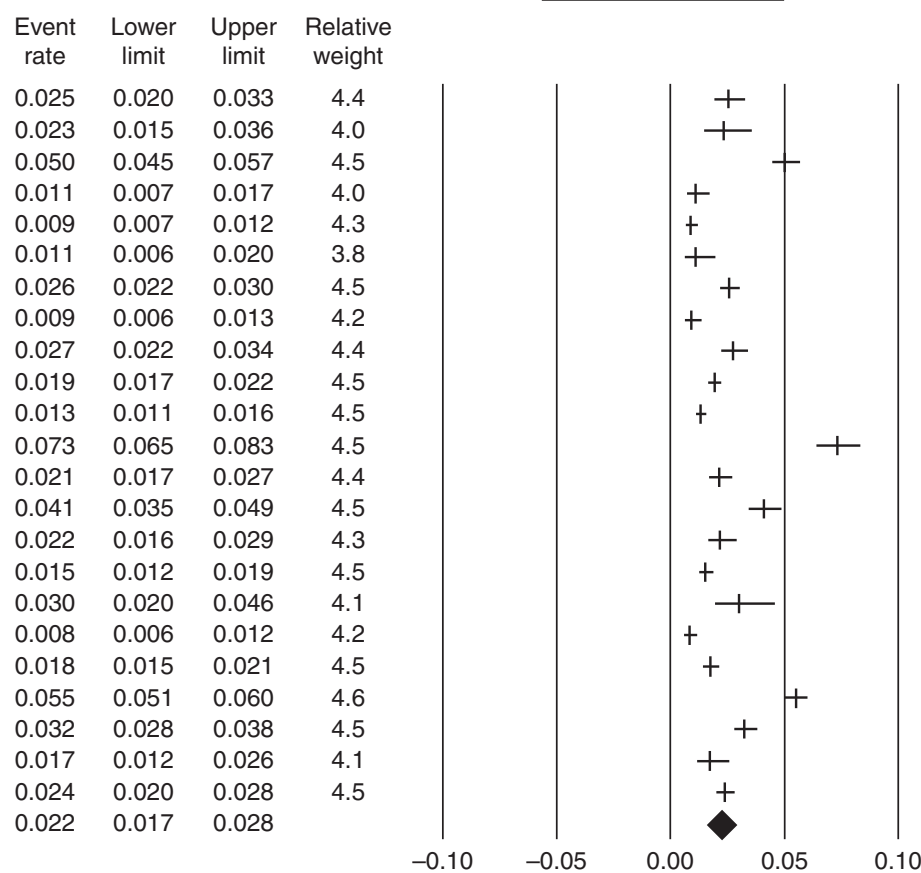

Figure 2. Proportion of proximal neoplasia (PN) and advanced proximal neoplasia (APN) in All Selected Studies (Random-Effect). (a) Proportion of proximal neoplasia (PN). (b) Proportion of advanced proximal neoplasia (APN). Cl, confidence interval.

with the findings by Lin et al. (9), demonstrating no increased risk of PN/APN for distal HP when compared with subjects who had normal distal findings. Our results imply that subjects with distal HP detected by FS should not be automatically referred for subsequent colonoscopy workup. Yet the findings of the present study should be interpreted with caution, as there is still a risk of PN/APN in subjects with normal distal colon or distal HPs-and proximal lesions could only be detected by colonoscopy. This is reflected by the pooled prevalence of isolated PN (5.6\%) and isolated APN (1.0\%), which could be regarded by some as significant and should be taken into account when one considers arrangement of follow-up colonoscopy.

Association studies between distal lesions and PN are important, given FS can only visualize the distal colon. The meta-analysis performed by Dodou and De Winter (10) found that the higher the histological grade of the distal finding, the higher the risk for both 
PN and APN. Our study presented a similar result, with relatively lower ORs. The major difference in the findings between this study and the meta-analysis by Dodou and De Winter (10) in 2012 could be attributed to a number of differences in study design. First, we have included studies that exclusively examined asymptomatic individuals as CRC screening participants. In addition, our metaanalysis included a much larger number of individuals, consisting of seven additional studies that were published after 2011-2012 (11-16,46), and one study performed in China with original data derived from high-quality colonoscopy procedures. Also, this meta-analysis has focused on the general screening population and excluded studies that evaluated the association between distal and proximal lesions among high-risk individuals that could potentially influence the magnitude of associations.

Proximal shift of CRC and increasing isolated PN have been reported in recent decades $(10,48,49)$. Nevertheless, identification of the association between the distal and proximal colon is particularly valuable in countries where colonoscopic capacity might be limited. This is especially the case as FS is increasingly used in some countries, including several European nations (50). The prediction for PN and APN is crucial not only for allocation of colonoscopy resource in population-based screening programs but also for tailoring screening option to reduce avoidable procedures, minimize unnecessary complications, and reducing health-care cost. A few prediction models for APN have been devised and validated (51); however, those models usually required many variables, and their discriminatory capability was fair. Several studies employed distal finding as predictors in their risk algorithms for APN $(12,31)$. For instance, Imperiale et al. (31) included age, gender, and distal finding as predictors-and the model achieved good internal validation $(c$-statistics $=0.74)$ with high discrimination. Park et al. (12) employed age, gender, smoking status, and distal finding detected by FS as predictive factors in an APN risk model for colonoscopy referral among low-risk subjects. This strategy might be more discriminatory than relying on FS result alone to risk-stratify subjects for colonoscopy in current practice protocols, because of their limited performance to predict APN $(13,15)$. The results of the present study are applicable to subjects who have undergone FS screening, where distal findings are available as a predictor for PN/APN.

For CRC screening, opportunistic testing for individuals is now shifting toward organized population screening program with high quality-control measures and regular surveillance intervals. Screening based on population-based design could be more representative of real-life practices in organized government programs, and the ORs of different distal findings to APN and PN in asymptomatic individuals retrieved from studies in such programs might be more generalizable. On the contrary, opportunistic recruitment of asymptomatic subjects who received colonoscopy might include subjects with more diverse risk profile (39). Therefore, stronger associations between distal findings and APN were observed in opportunistic screening design with higher degrees of heterogeneity. The OR for AN-APN and AN-PN in studies that adopted population-based designs could be more representative of real clinical practice. Endoscopy quality, among all quality-control measures to ensure high-quality CRC screening programs, is another important effect modifier. It is noticed that the OR of distal AN for APN in the studies with less stringent endoscopy quality control were higher than that in studies with more stringent quality-control procedures. The explanation of these findings remains to be explored in future studies.

When serrated lesions were included in the definition of $\mathrm{AN}$, it was observed that the magnitude of the OR was higher; the difference was, however, not statistically significant as there were only two studies that included serrated lesions. A previous study that examined a large cohort of Chinese screening participants found that the presence of large and proximal serrated polyps was an independent risk factor for synchronous advanced colorectal

Table 3. Proportion and OR of PN/APN among subjects of different distal findings (random effects)

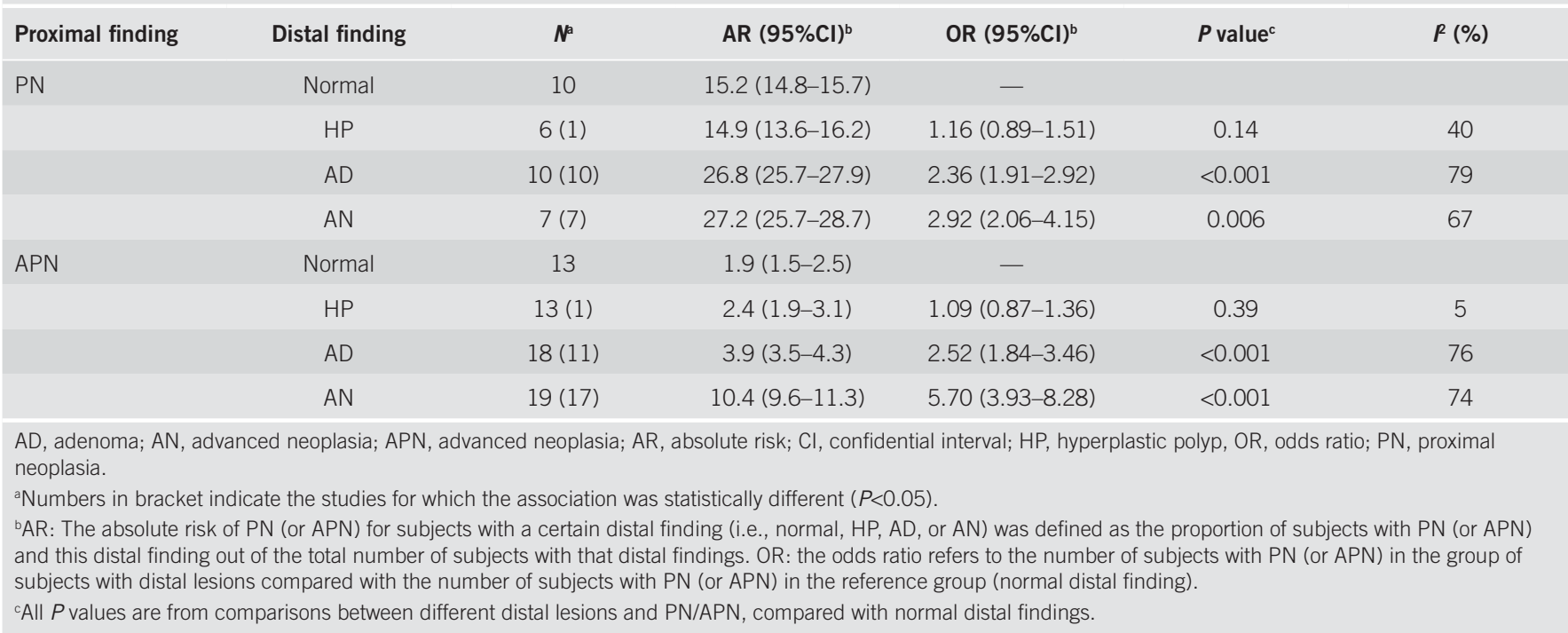


neoplasia and multiple non-AN. Hence, the association between proximal and distal lesions might be different when serrated lesions were included as they were considered as markers of more advanced colonic lesions (52). In addition, the definition of "distal" was also found to modify the association between APN and distal AN. Lesions detected in the descending colon and splenic flexure may represent more advanced serrated lesion and possibly serrated polyposis syndrome, in which multiple serrated and HPs were detected in the whole colon. Furthermore, analyses including studies with FS followed by a subsequent colonoscopy as a separate procedure (vs. colonoscopy alone) generated weaker associations between proximal and distal lesions. This observation might be attributed to the differences in bowel preparation, endoscopic procedural factors, and the possible involvement of two or more endoscopists for the former group. As these subgroup analyses included small number of studies and sample size, future evaluation of these associations by larger-scale studies is required.

Our meta-analysis has a number of strengths. First, it included asymptomatic, average-risk subjects in all selected studies. Hence, the application of its findings is more generalizable to screening practices when compared with previous meta-analyses. Also, it is the most updated meta-analysis with the largest number of screening participants included from all published studies. In addition, various moderators of the association between proximal and distal findings were addressed in subgroup analyses. We performed a quality assessment based on an internationally recognized Newcastle Ottawa Scale scale for all the selected articles in a systematic manner. Nevertheless, some limitations should be mentioned. For instance, publication bias might exist and we could have missed some gray literature or informal reports. In addition, the bowel preparation quality and adenoma detection rate, as well as the qualification and experience of endoscopists involved in the FS and colonoscopy procedures, might be different, while most of the studies performed colonoscopy to simulate a procedure where FS was followed by colonoscopy. Even though patients might not have a risk of PN detected if the colonoscopy was performed at around the same time as the FS, there could be a risk of PN when the patients receive colonoscopy at a significantly later time period. Also, larger and more numerous distal HPs could potentially indicate higher risk, such as the presence of serrated

a

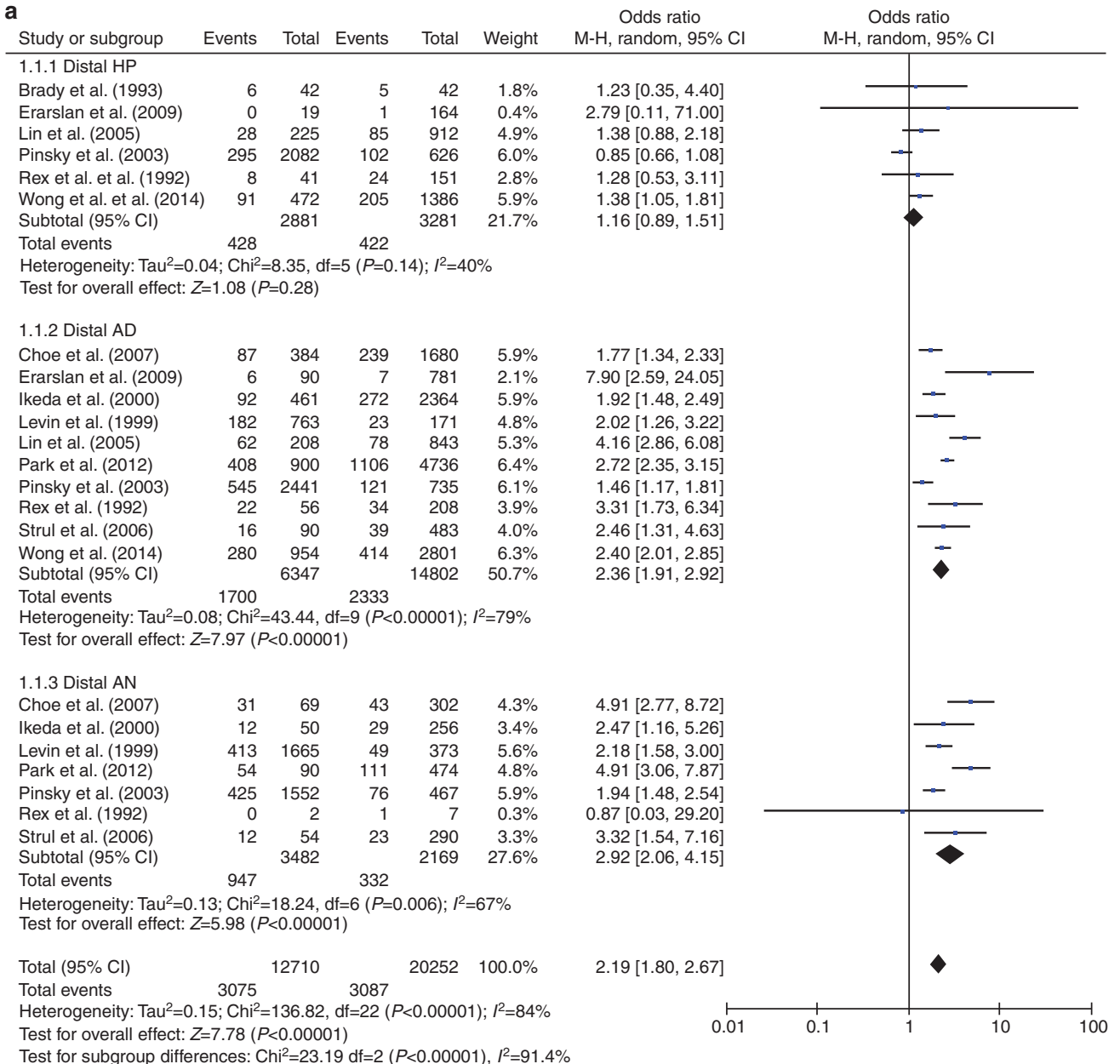

Figure 3. Forest plots of the association between distal findings (hyperplstic polyp (HP), adenoma (AD), advanced neoplasia (AN)) and PN/APN comparing with normal distal findings (Random-Effect). (a) Odds of proximal neoplasia (PN). (b) Odds of advanced proximal neoplasia (APN). Cl, confidence interval. 


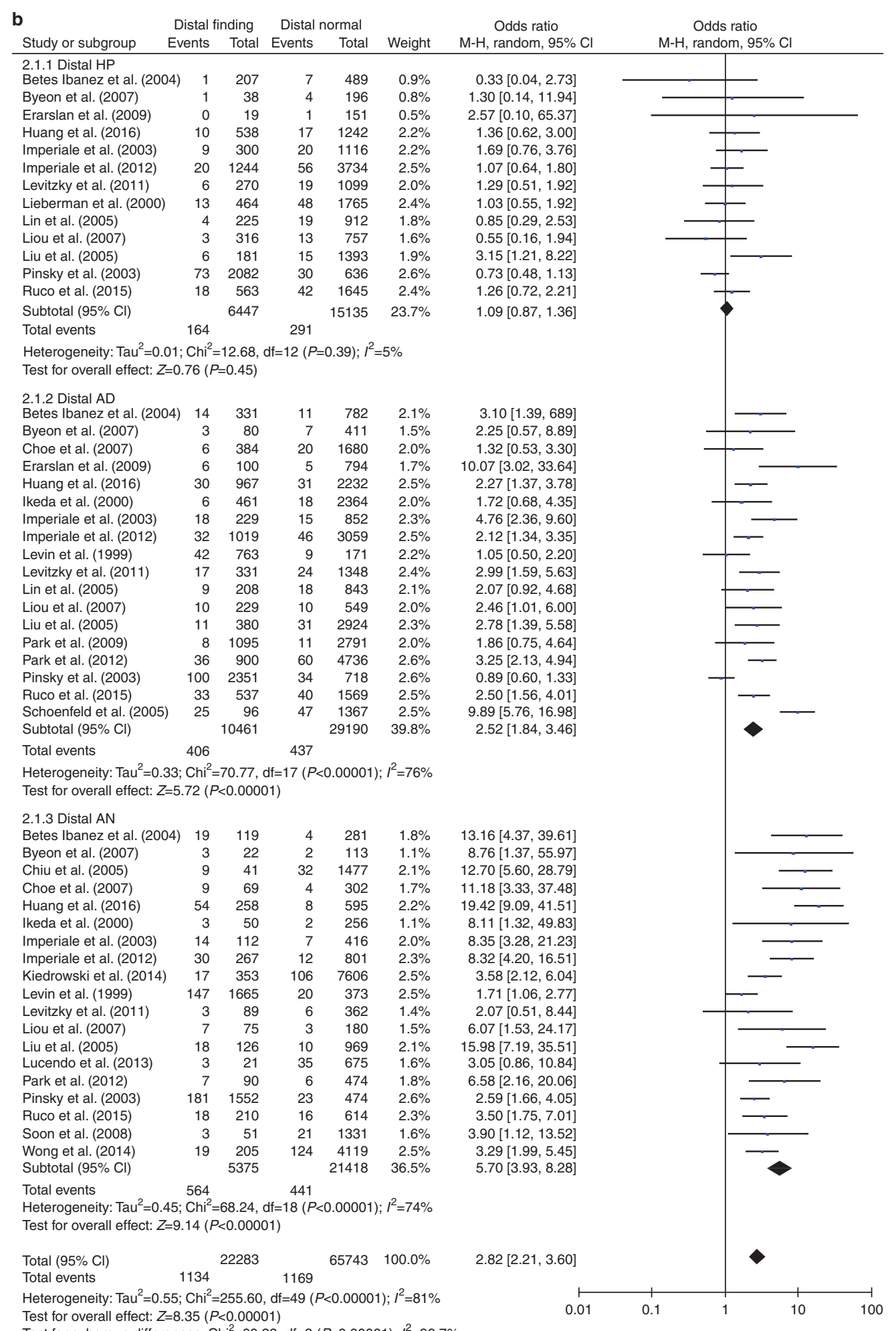

Figure 3. Continued.

polyposis syndrome; yet none of the primary studies consisted of data on the size and number of distal HPs nor the different subtypes of serrated lesions. Finally, it is well recognized that the prevalence and distribution of colorectal neoplasia varied in people of different ethnicities, yet the present studies recruited subjects from America, Europe, and Asia only. The heterogeneity of the study findings could be partly accounted for by the pooled ORs from screening participants of different ethnicities. 
Table 4. Subgroup analysis according to study characteristics

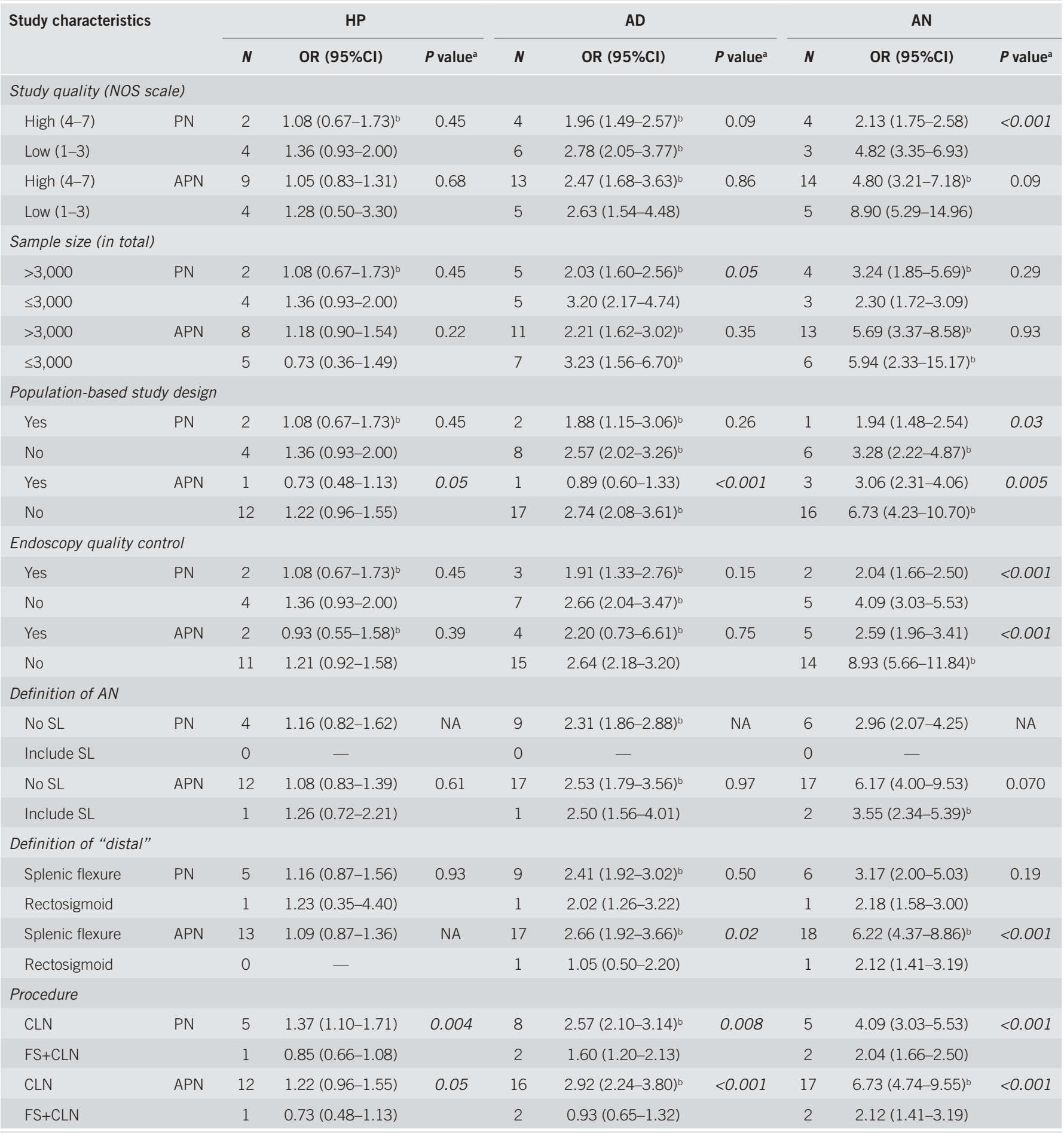

AD, adenoma; AN, advanced neoplasia; APN, advanced proximal neoplasia; Cl, confidential interval; CLN, colonoscopy; FS, flexible sigmoidoscopy; HP, hyperplastic polyp, NOS, Newcastle Ottawa Scale; OR, odds ratio; PN, proximal neoplasia; SL, serrated lesions. If $p<0.05$, the difference between the two subgroups is statistically significant.

a $P$ values refer to the Cochran tests for differences between subgroups.

${ }^{\mathrm{b}} P<0.05$ in heterogeneity test. 
In conclusion, distal HP is neither a marker for PN nor APN in asymptomatic screening population when compared with normal distal findings. The ORs of AD and distal AN for PN/APN were significantly increased. These findings did not support routine referral of all subjects detected having distal HPs. We anticipate that this clinical implication has a substantial potential to reduce unnecessary colonoscopy procedure, complications, and health-care costs. Future prospective studies employing population-based design including screening participants of different ethnicities screened by good quality-control endoscopies could shed more light on the relationship between distal and proximal findings.

\section{ACKNOWLEDGMENTS}

We wish to express our gratitude to the statistical advice from the Division of Biostatistics of the School of Public Health and Primary Care, Chinese University of Hong Kong.

\section{CONFLICT OF INTEREST}

Guarantor of the article: Jason L.W. Huang, MD.

Specific author contributions: J.L.W.H., Y.H.W., and M.C.S.W. had full access to all the data in the study and take responsibility for the data and the accuracy of the data analysis. M.C.S.W., J.L.W.H., Y.H.W., and J.Y.J. contributed to the study concept and design. C.P.Y. offered advice on search strategy. All authors analyzed and interpreted the data. J.L.W.H., H.H.X.W., and J.Y.J. critically revised the manuscript. All authors read and approved the final manuscript. All authors included on the paper fulfill the criteria of authorship. There is no one else who fulfills the criteria but has not been included as an author. Financial Support: None.

Potential Competing Interests: None.

\section{Study Highlights}

\section{WHAT IS CURRENT KNOWLEDGE}

Flexible sigmoidoscopy (FS) becomes more popular as a primary screening test for colorectal cancer (CRC) screening in some European and Asian countries.

FS is a recommended test of choice designed both to detect and prevent CRC if colonoscopy is not available or acceptable to patients.

Whether subjects with distal hyperplastic polyps (HPs) detected should be followed up by colonoscopy workup remains controversial.

\section{WHAT IS NEW HERE}

A meta-analysis including 28 studies that included 104,961 average-risk asymptomatic participants for colorectal cancer screening was conducted.

When compared with normal distal findings, distal HP was not associated with proximal neoplasia (PN) or advanced proximal neoplasia (APN).

Subjects with distal non-advanced or advanced adenoma had higher odds of PN/APN, and higher odds of PN/APN were observed for more severe distal lesions.

\section{REFERENCES}

1. Brenner H, Kloor M, Pox CP.. Colorectal cancer. Lancet 2014;383: 1490-502.

2. Sung JJY, Ng SC, Chan FK et al. An updated Asia Pacific Consensus Recommendations on colorectal cancer screening. Gut 2015;64:121-32.

3. Leddin DJ.. Canadian Association of Gastroenterology position statement on screening individuals at average risk for developing colorectal cancer: 2010. Can J Gastroenterol 2010;24:705-14.

4. Qaseem A, Denberg TD, Hopkins RH et al. Screening for colorectal cancer: a guidance statement from the American College of Physicians. Ann Intern Med 2012;156:378-86.

5. Bibbins-Domingo K, Grossman DC et al. US Preventive Services Task Force. Screening for colorectal cancer: US Preventive Services Task Force recommendation statement. JAMA 2016;315:2564-75.

6. Lin JS, Piper MA, Perdue LA et al. Screening for colorectal cancer: updated evidence report and systematic review for the US Preventive Services Task Force. JAMA 2016;315:3576-94.

7. Dave S, Hui S, Kroenke K et al. Is the distal hyperplastic polyp a marker for proximal neoplasia? A systematic review. J Gen Intern Med 2003;18: $128-37$.

8. Lewis JD, $\mathrm{Ng} \mathrm{K}$, Hung KE et al. Detection of proximal adenomatous polyps with screening sigmoidoscopy: a systematic review and meta-analysis of screening colonoscopy. Arch Intern Med 2003;163:413-20.

9. Lin OS, Gerson LB, Soon MS et al. Risk of proximal colon neoplasia with distal hyperplastic polyps: A meta-analysis. Arch Intern Med 2005;165: 382-90.

10. Dodou D, De Winter JC. The relationship between distal and proximal colonic neoplasia: a meta-analysis. J Gen Intern Med 2012;27:361-70.

11. Imperiale TF, Glowinski EA, Lin-Cooper C et al. Tailoring colorectal cancer screening by considering risk of advanced proximal neoplasia. Am J Med 2012;125:1181-7.

12. Park HW, Han S, Lee JS et al. Risk stratification for advanced proximal colon neoplasm and individualized endoscopic screening for colorectal cancer by a risk-scoring model. Gastrointest Endosc 2012;76:818-28.

13. Castells A, Bessa X, Quintero E et al. Risk of advanced proximal neoplasms according to distal colorectal findings: comparison of sigmoidoscopy-based strategies. J Natl Cancer Inst 2013;105:878-86.

14. Kiedrowski M, Mroz A, Kaminski MF et al. Predictive factors of proximal advanced neoplasia in the large bowel. Arch Med Sci 2014;10:484-9.

15. Wong MCS, Ching JYL, Ng SC et al. Prediction of proximal advanced neoplasia: A comparison of four existing sigmoidoscopy-based strategies in a Chinese population. Gut 2014;64:776-83.

16. Ruco A, Stock D, Hilsden RJ et al. Evaluation of a risk index for advanced proximal neoplasia of the colon. Gastrointest Endosc 2015;81:1427-32.

17. Liberati A, Altman DG, Tetzlaff J et al. The PRISMA statement for reporting systematic reviews and meta-analyses of studies that evaluate health care interventions: explanation and elaboration. Ann Intern Med 2009;151:W65-94.

18. Gupta S, Balasubramanian BA, Fu T et al. Polyps with advanced neoplasia are smaller in the right than in the left colon: implications for colorectal cancer screening. Clin Gastroenterol Hepatol 2012;10:1395-401.e2

19. Parra-Pérez V, Watanabe-Yamamoto J, Nago-Nago A et al. Factors related to advanced colorectal neoplasm at the Policlínico PeruanoJaponés. Rev Gastroenterol Mex 2015;80:239-47.

20. Lieberman DA, Prindiville S, Weiss DG et al. VA Cooperative Study Group 380. Risk factors for advanced colonic neoplasia and hyperplastic polyps in asymptomatic individuals. JAMA 2003;290:2959-67.

21. Foutch PG, Mai H, Pardy K et al. Flexible sigmoidoscopy may be ineffective for secondary prevention of colorectal cancer in asymptomatic, average-risk men. Dig Dis Sci 1991;36:924-8.

22. Thiis-Evensen E., Hoff GS, Sauar J et al. Flexible sigmoidoscopy or colonoscopy as a screening modality for colorectal adenomas in older age groups? Findings in a cohort of the normal population aged $63-72$ years. Gut 1999;45:834.

23. Gryska P, Cohen A.. Screening asymptomatic patients at high risk for colon cancer with full colonoscopy. Dis Colon Rectum 1987;30:18-20.

24. Kahi CJ, Vemulapalli KC, Snover D et al. Distal colorectal findings are not related to the risk of proximal advanced serrated neoplasia. Digestive Diease Week 03-06 May 2014, Vol. 79 ASGE, Chicago, IL, USA, 2014, pp. AB421.

25. Herzog R, Álvarez-Pasquin MJ, Díaz C et al. Are healthcare workers' intentions to vaccinate related to their knowledge, beliefs and attitudes? A systematic review. BMC Public Health 2013;13:154. 
26. Rex DK, Smith JJ, Ulbright TM et al. Distal colonic hyperplastic polyps do not predict proximal adenomas in asymptomatic average-risk subjects. Gastroenterology 1992;102:317-9.

27. Brady PG, Straker RJ, McClave SA et al. Are hyperplastic rectosigmoid polyps associated with an increased risk of proximal colonic neoplasms? Gastrointest Endosc 1993;39:481-5.

28. Levin TR, Palitz A, Grossman S et al. Predicting advanced proximal colonic neoplasia with screening sigmoidoscopy. J Am Med Assoc 1999;281: 1611-7.

29. Ikeda Y, Mori M, Miyazaki M et al. Significance of small distal adenoma for detection of proximal neoplasms in the colorectum. Gastrointest Endosc 2000;52:358-61.

30. Lieberman DA, Weiss DG, Bond JH et al. Use of colonoscopy to screen asymptomatic adults for colorectal cancer. N Engl J Med 2000;343: $162-8$.

31. Imperiale TF, Wagner DR, Lin CY et al. Using risk for advanced proximal colonic neoplasia to tailor endoscopic screening for colorectal cancer. Ann Intern Med 2003;139:959-96510.

32. Pinsky PF, Schoen RE, Weissfeld JL et al. Predictors of advanced proximal neoplasia in persons with abnormal screening flexible sigmoidoscopy. Clin Gastroenterol Hepatol. 2003 1:103-10.

33. Betés Ibáñez M, Munoz-Navas MA, Duque JM et al. Diagnostic value of distal colonic polyps for prediction of advanced proximal neoplasia in an average-risk population undergoing screening colonoscopy. Gastrointest Endosc. 2004 59:634-41.

34. Chiu HM, Wang HP, Lee YC et al. A prospective study of the frequency and the topographical distribution of colon neoplasia in asymptomatic averagerisk Chinese adults as determined by colonoscopic screening. Gastrointest Endosc 2005;61:547-53.

35. Lin OS, Schembre DB, McCormick SE et al. Risk of proximal colorectal neoplasia among asymptomatic patients with distal hyperplastic polyps. Am J Med 2005;118:1113-9.

36. Liu HH, Wu MC, Peng Y et al. Prevalence of advanced colonic polyps in asymptomatic Chinese. World J Gastroenterol 2005;11:4731-4.

37. Schoenfeld P, Cash B, Flood A et al. Colonoscopic screening of average-risk women for colorectal neoplasia. N Engl J Med 2005;352:2061-8.

38. Strul H, Kariv R, Leshno $\mathrm{M}$ et al. The prevalence rate and anatomic location of colorectal adenoma and cancer detected by colonoscopy in average-risk individuals aged 40-80 years. Am J Gastroenterol 2006;101:255-62.

39. Byeon J, Yang S, Kim TI et al. Colorectal neoplasm in asymptomatic Asians: a prospective multinational multicenter colonoscopy survey. Gastrointest Endosc 2007;65:1015-22.

40. Choe JW, Chang H, Yang S et al. Screening colonoscopy in asymptomatic average-risk Koreans: analysis in relation to age and sex. J Gastroenterol Hepatol 2007;22:1003-8.

41. Liou JM, Lin JT, Huang SP et al. Screening for colorectal cancer in averagerisk Chinese population using a mixed strategy with sigmoidoscopy and colonoscopy. Dis Colon Rectum 2007;50:630-40.
42. Soon M, Soon A, Lin T et al. Distribution of colon neoplasia in Chinese patients: implications for endoscopic screening strategies. Eur J Gastroenterol Hepatol 2008;20:642-7.

43. Erarslan E, Turkay C, Isik A et al. Prevalence of proximal neoplasms among asymptomatic patients according to distal colorectal findings. Dig Dis Sci 2009;54:1312-6.

44. Park H-, Byeon J-, Yang S- et al. Colorectal neoplasm in asymptomatic average-Risk Koreans: the KASID prospective multicenter colonoscopy survey. Gut Liver 2009;3:35-40

45. Levitzky BE, Brown CC, Heeren TC et al. Performance of a risk index for advanced proximal colorectal neoplasia among a racially/ethnically diverse patient population (risk index for advanced proximal neoplasia). Am J Gastroenterol 2011;106:1099-106.

46. Lucendo AJ, Guagnozzi D, Angueira T et al. The relationship between proximal and distal colonic adenomas: is screening sigmoidoscopy enough in the presence of a changing epidemiology? Eur J Gastroenterol Hepatol 2013;25:973-80.

47. Huang JL, Chen P, Yuan X. An algorithm to predict advanced proximal colorectal neoplasia in Chinese asymptomatic population. Scientific Reports 2017;7; doi:10.1038/srep46493.

48. McCallion K, Mitchell RMS, Wilson RH et al. Flexible sigmoidoscopy and the changing distribution of colorectal cancer: Implications for screening. Gut 2001;48:522-5.

49. Rozen P, Liphshitz I, Barchana M.. The changing epidemiology of colorectal cancer and its relevance for adapting screening guidelines and methods. Eur J Cancer Prev 2011;20:46-53

50. Schreuders EH, Ruco A, Rabeneck L et al. Colorectal cancer screening: a global overview of existing programmes. Gut 2015;64:1637-49.

51. Imperiale TF, Monahan PO, Stump TE et al. Predicting advanced proximal neoplasia in asymptomatic adults without knowing distal colorectal findings: a new scoring system with high discrimination. Digestive Disease Week 17-19 May, 2015, Vol. 148, Washington, DC, USA, 2015, pp. S780.

52. Ng SC, Ching JY, Chan VC et al. Association between serrated polyps and the risk of synchronous advanced colorectal neoplasia in average-risk individuals. Aliment PharmacolTher 2015;41:108-15.

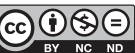

This work is licensed under a Creative Commons Attribution-NonCommercial-NoDerivs 4.0 International License. The images or other third party material in this article are included in the article's Creative Commons license, unless indicated otherwise in the credit line; if the material is not included under the Creative Commons license, users will need to obtain permission from the license holder to reproduce the material. To view a copy of this license, visit http://creativecommons.org/licenses/by-nc-nd/4.0/

(C) The Author(s) 2017 